\title{
THE SCOPE AND LIMITATIONS OF VISUAL AND AUTOMATIC ANALYSIS OF THE ELECTROENCEPHALOGRAM
}

\author{
BY
}

\author{
G. D. DAWSON
}

(The David Lewis Epileptic Colony)

and

\section{W. GREY WALTER}

(The Burden Neurological Institute)

(RECEIVED 10TH November, 1944)

\begin{abstract}
Introduction
SINCE early in the history of the electroencephalogram (e.e.g.) it has been realized that the records are complex. That is to say, the rhythms in them are rarely simple harmonic oscillations and often have a number of components. The component oscillations may either bear some constant harmonic relation to one another or they may be entirely independent in their frequencies. The formation of complex waves from a number of simple sinusoidal components obeys certain well established physical principles. The purpose of this paper is to consider how these principles must affect the interpretation of an e.e.g. record. From this consideration two suggestions will result; first, that an application of the principles to the visual inspection and measurement of a record can materially increase the amount of information obtainable, and secondly, that there are certain types of change that occur in the e.e.g. that cannot be measured and assessed accurately except by a more elaborate form of analysis.
\end{abstract}

Analysis of the e.e.g. to separate its components has been made in the past in a number of ways. Mathematical analysis of a short length of record (Rohracher, 1937; Livanov and Petrova, 1938) is exceedingly tedious and not applicable to routine work. This method may, however, be of value in special cases, as it may give a degree of accuracy limited only by the quality of the original recording. Selective recording methods (Drohocki, 1938; Davis et al. 1938; Parr and Walter, 1943) are limited in scope as only a restricted part of the e.e.g. frequency band may be studied at one time. Until recently the only complete, accurate, and practical method described has been that due to Grass (Grass and Gibbs, 1938). In this method a special photographic record is made, a part of which is selected corresponding to thirty seconds of recording. This length of record is then analysed by an automatic electrical apparatus. A high degree of accuracy is obtained and the method has contributed much valuable information about the changes in the e.e.g. occurring in different physiological and pathological states. Unfortunately the length of record that may be analysed has to be limited. This, together with the need of a special record, probably accounts for the failure of this method to be readily accepted as a routine.

Recently a method has been described in which every ten seconds of record is analysed (Walter, $1943 a$ and $b$ ). The analysis is automatically traced alongside the record in the form of a histogram, within ten seconds of the recording of the e.e.g. No extra labour is involved during or after the examination, and analysis during the entire period of recording becomes possible as a routine. It therefore seems desirable to decide what are the limitations of a visual inspection and measurement of an e.e.g record and when this fuller analysis is essential.

Study of a mixture of rhythms, such as make up the e.e.g., is difficult on account of the fact that the components rarely remain constant for long. To overcome this difficulty, and to demonstrate in a pure form the appearances that are produced when a number of rhythms are mixed, a method of synthesis of compound waveforms has been adopted. The method is an electro-mechanical one which allows a rapid alteration to be made in the frequency or amplitude of one or more of the components. By this means it is possible to mimic accurately the various waveforms that occur in the e.e.g. The ability to produce these effects without the distractions of the rapid changes occurring in the natural e.e.g., has proved of great value in visualizing the way in which a given wave in the e.e.g. may be expected to change in altered physiological conditions. In addition, a useful check on the accuracy of an analysis of the e.e.g. is available, for a synthesis of the components indicated, in the correct amounts, should in most cases produce a wave identical with that analysed.

Here it should be made clear that a synthesis of 
this kind may equally well be made by graphical methods, and that for many years this has in fact been done for other purposes. An example of the valuable information that graphic synthesis may yield is given by the work of Gasser and Erlanger (1927) who used it to determine the components of the complex action potential wave in nerve. The graphic form of synthesis is, however, exceedingly tedious and is nei practical as a demonstration method.

\section{Method}

Two or more oscillators giving simple harmonic oscillations of the same order of size and frequency as occur in the e.e.g. are used. These oscillators are connected to the inputs of a standard e.e.g. apparatus in such a way that one channel records the algebraical sum of the amplitudes of the oscillations and the other channels record the individual oscillations separately. Changes in a component and the resultant may thus be seen at the same time.

The oscillators used are of the photomechanical type. A selenium barrier-layer photoelectric cell is illuminated from a light source of fixed intensity and a suitably slotted disc interposed between the light and the cell. If the disc is rotated and the slots have the correct shape the amount of light falling on the cell will vary in a sinusoidal manner and the cell will produce a potential difference of sinewave form. The size of the potential difference may be altered by a potentiometer connected across the cell and the frequency of the oscillation changed by altering the rate of rotation of the disc. Oscillators using the photoelectric principle, also using capacities varied in size mechanically, have been described before (Schaffer and Lubsinski, 1931; Kurtz and Larsen, 1935). More recently an electronic method, suitable for use in the audible sound frequency band from 50 to $20,000 \mathrm{c}$./ $/ \mathrm{sec}$., but unsuitable for use at the low frequencies at which an e.e.g. apparatus works, has been described (Somerville, 1944).

In the earlier experiments when two or three separate oscillators have been useri, some difficulty has been experienced in keeping them accurately in step, if constant frequency relations between several waves have been required. The results, however, have been so enlightening that a more complicated apparatus has been made. This apparatus, which is to be described elsewhere (Walter and Baldock, in press), will produce a harmonically related series of ten oscillations simultaneously. The individual generators are locked together on a common spindle and the frequency relations and phases of the oscillations may be adjusted and held absolutely constant.

\section{Terminology}

In the following description of compound waves it is assumed that the meaning of the terms Amplitude, Frequency, Cycle, and Sinewave oscillation are understood. For the last of these the terms simple harmonic oscillation and sinusoidal oscillation are also used. Other terms used may be less familiar and the sense in which they are used will be defined.

Harmonic Series.-When a series of sinusoidal oscillations have frequencies that are whole multiples of that of a single oscillation, which is called the fundamental, they compose part of a harmonic series. Thus, in a series in which the fundamental, or first harmonic, is 1 cycle per second $(1 \mathrm{c} . / \mathrm{sec}$.$) ,$ the second harmonic will have a frequency of $2 \mathrm{c} . / \mathrm{sec}$., the third of $3 \mathrm{c}$. $/ \mathrm{sec}$., and so on. In a series with the fundamental $2 \mathrm{c}$./sec., frequencies of $4 \mathrm{c}$. $/ \mathrm{sec}$. and $6 \mathrm{c}$. $/ \mathrm{sec}$. would be called the second and third harmonics of the fundamental. The series need not necessarily be complete, any number of terms may be missing. For instance, two oscillations of frequency 20 and $22 \mathrm{c}$./sec. are the tenth and eleventh terms in a series with a fundamental of zero amplitude at $2 \mathrm{c}$. $/ \mathrm{sec}$.

Phase.-The phase of an oscillation at any instant is considered to be that part of a complete cycle that has occurred since any arbitrarily chosen point in the cycle of oscillation. This is usually expressed as an angle; one complete cycle being $360^{\circ}$. Thus, if the zero position of a sinewave is taken as reference the oscillation is said to have completed $90^{\circ}$ when the first peak is reached. Two oscillations are said to be in phase when they are passing through the same part of a cycle at the same time. When the peak of one oscillation coincides with zero of another they are $90^{\circ}$ out of phase; when both oscillations leave their zero positions simultaneously, but in opposite directions, they are $180^{\circ}$ out of phase.

Modulation.-When a single oscillation undergoes a rhythmical change in size it is said to be modulated in amplitude. If the amplitude is made to fluctuate between zero and twice the unmodulated size, the modulation is said to be 100 per cent. The percentage modulation is therefore an expression of the amount of fluctuation above and below a mean amplitude. For example, a 50 per cent. modulated wave would vary by plus and minus 50 per cent. of its mean amplitude.

Beats.-If the amplitudes of two sinusoidal oscillations are summed algebraically, the resultant will fluctuate in size at a rate equal to the difference in the frequency of the components. These periodic maxima and minima, which are called beats, are most obvious when the difference is a relatively small percentage of the frequencies of the components. The reason for the beating may be seen if two oscillations of $5 \mathrm{c}$./sec. and $6 \mathrm{c}$./sec. are considered. If these two oscillations have the same initial phase the sum of their amplitudes will be greater than that of either alone at this time. At the end of half a second, however, the $6 \mathrm{c}$. $/ \mathrm{sec}$. oscillation will have completed 3 cycles; the $5 \mathrm{c}$./ $/ \mathrm{sec}$. oscillation will have completed only 2.5 cycles. The oscillations at this point will therefore be in opposite phase, and the amplitude of the resultant will be at a minimum. At the end of the full second, the cycle will be complete, and the waves will be in phase again. In the special case where the two oscillations have equal amplitudes, the resultant will fluctuate between zero and twice the size of either oscillation alone. Therefore, beating will always be most marked when the amplitudes of the two oscillations are nearly equal.

\section{Results}

A feature of certain types of e.e.g. record is the apparent cessation of a rhythm of 4-7 c./sec., in the parieto-occipital regions, when the alpha rhythm is produced on closing the eyes. This series of 
events has been interpreted in two ways. Usually it has been considered that the 4-7 c./sec. rhythm has either speeded up to the faster rate and is itself a slow form of the alpha rhythm, or that the occurrence of the alpha rhythm inhibits the slower rhythm.

Analysis of the records, during the periods when the alpha rhythm is present and the slower rhythm apparently absent, gives an interesting result. It shows that in many cases the slow rhythm is quite unaltered in size, although it may not be seen in the record. This effect will be referred to as masking, and a rhythm which is shown to be present in a record by analysis, but which cannot be seen in the primary record, will be referred to as being masked.

To demonstrate this effect of masking of one rhythm by another, two rhythms, one of $8.5 \mathrm{c}$. $/ \mathrm{sec}$. and one of $6 \mathrm{c}$. $/ \mathrm{sec}$., have been combined in various sizes. The compound waves resulting are shown in Fig. 1. In (a) the two component rhythms are of almost equal amplitude, the résultant fluctuates in size from zero to twice the amplitude of either component, and the beats occur two and a half times a second. In $(b)$ the $6 \mathrm{c} . / \mathrm{sec}$. and $(d)$ the $8.5 \mathrm{c} . / \mathrm{sec}$. component is reduced in size by half. In $(c)$ and $(e)$ the same components respectively are reduced to a quarter of their original size. It immediately becomes obvious that the only frequency that may be counted directly in the resultant is that of the larger component.

When a rhythm, such as the resultant in Fig. 1 (b) is seen in an e.e.g. record its frequency, $8.5 \mathrm{c} . / \mathrm{sec}$., may be counted and the rate at which it fluctuates, 2.5 times a second, may be measured. The question then arises as to whether this fluctuation is due to the presence of a masked $6 \mathrm{c}$./sec. rhythm, or whether there is only an $8.5 \mathrm{c}$. $/ \mathrm{sec}$. rhythm that is modulated 2.5 times a second. Single rhythms, modulated by fluctuating excitability, or degrees of synchronization of brain cells may occur. The outline of a wave that is sinusoidally modulated and of the resultant of two rhythms beating together, are different. To show this difference in its most obvious form frequencies higher than those normally found in the e.e.g. are used. In Fig. 2 are shown the effects of modulating a $50 \mathrm{c} . / \mathrm{sec}$. rhythm at 1.25 times a second and of beating between a rhythm of $50 \mathrm{c}$./sec. and another of 48 to $49 \mathrm{c}$./ $/ \mathrm{sec}$. In the records of modulation, Fig. $2(a),(b)$, and (c), the top line indicates the extent and rate of modulation of the single frequency shown in the second line, and the third line shows the result of such modulation of the single frequency. The third line is not in this case a resultant of the algebraical summation of the top and second lines as is the case in the records of beating. The recording rate is such that while the individual waves

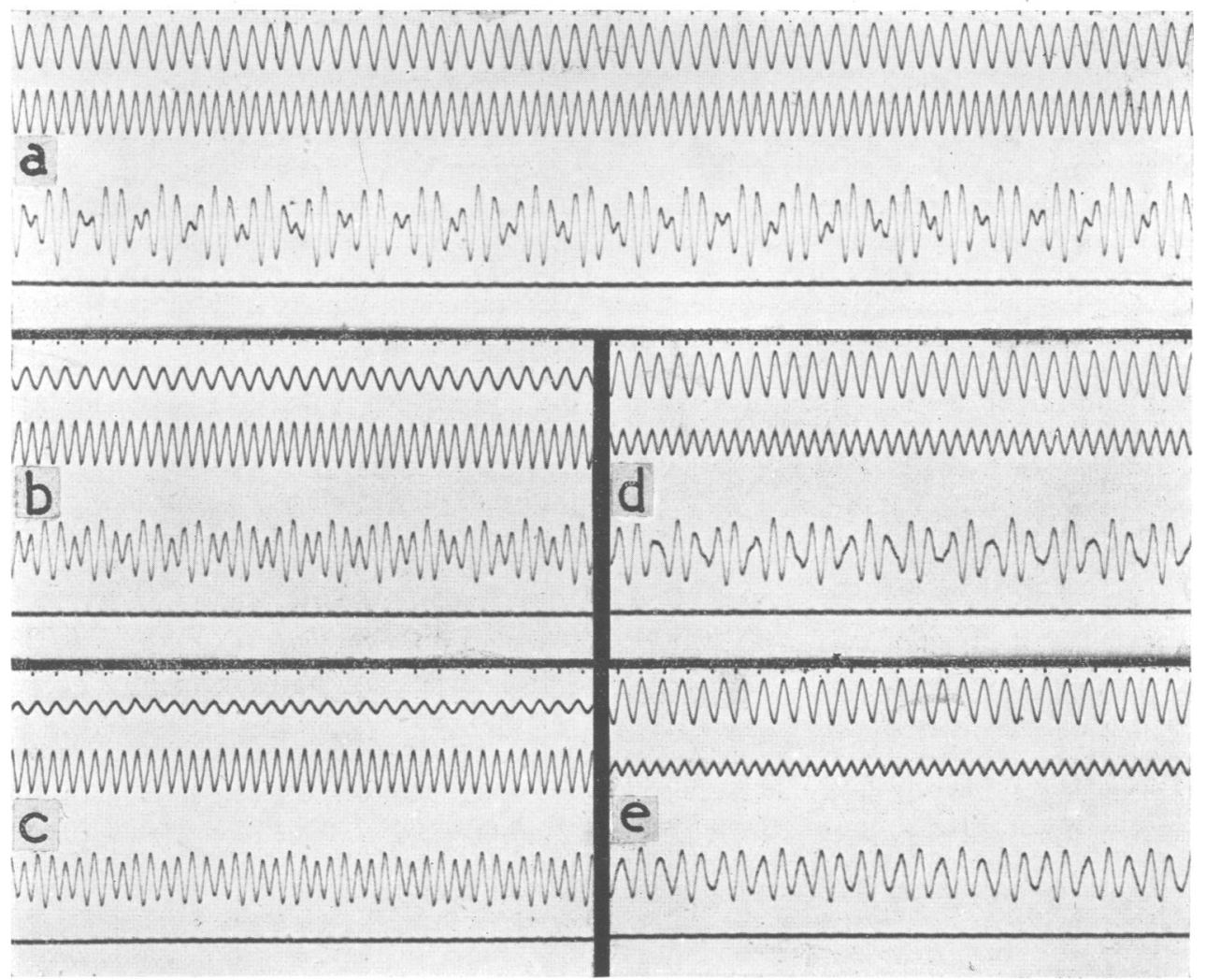

Fig. 1.-Cathode-ray oscillograph records showing masking of a smaller rhythm by a larger: (a) 8.5 c./sec. and $6 \mathrm{c}$./sec. equal; (b) $6 \mathrm{c}$./sec. reduced to 50 per cent.; (c) $6 \mathrm{c}$./sec. reduced to 25 per cent.; (d) $8.5 \mathrm{c}$. $/ \mathrm{sec}$. reduced to 50 per cent.; (e) $8.5 \mathrm{c}$./sec. reduced to 25 per cent. In each record the upper trace shows the $6 \mathrm{c}$. $/ \mathrm{sec}$. rhythm, the middle the $8.5 \mathrm{c}$./sec. rhythm, and the lowest the resultant of the two. Time marks at top of each record shows one-fifth sec. 


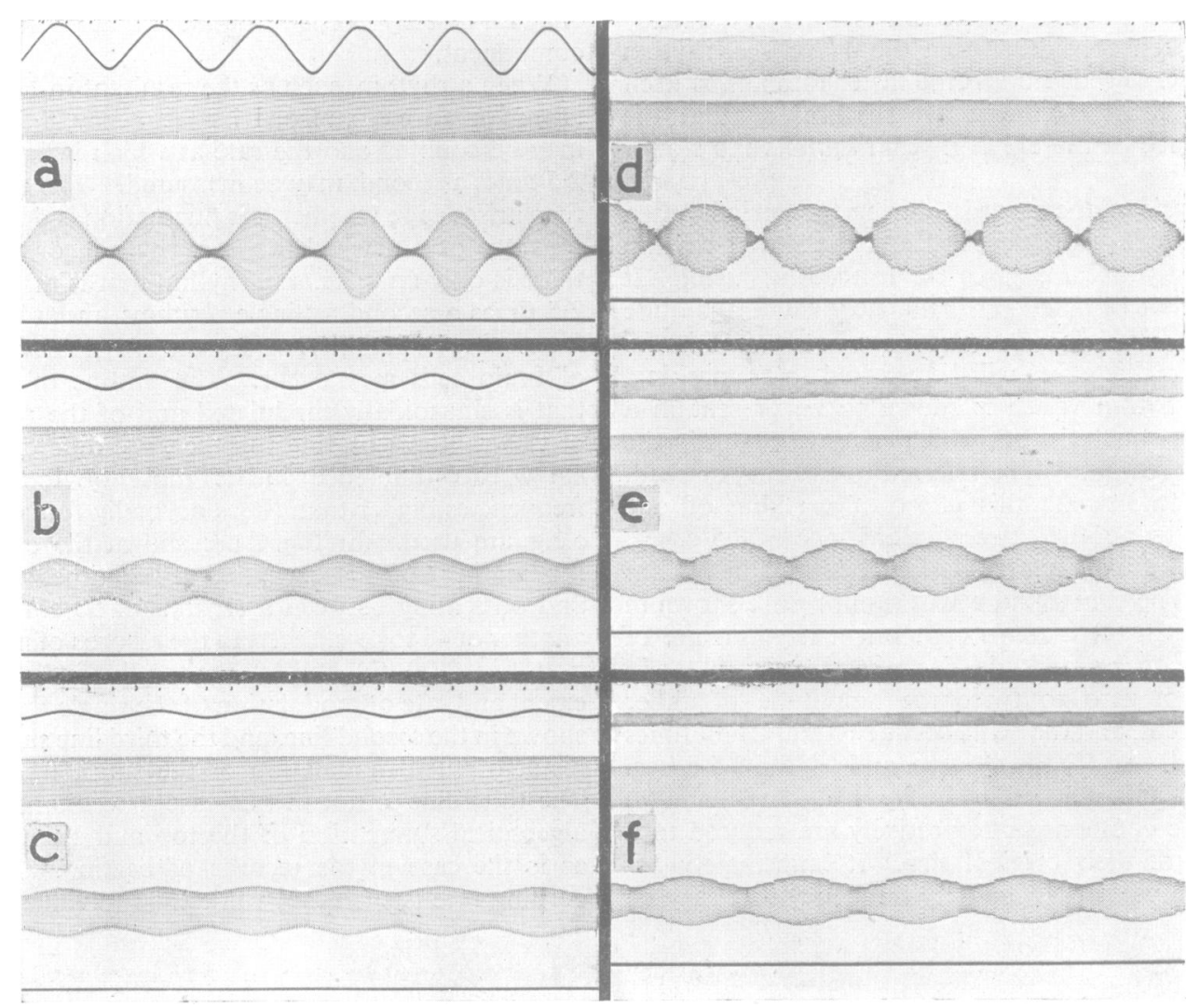

Fig. 2.-(a) 100 per cent. modulation of a $50 \mathrm{c}$ / $/ \mathrm{sec}$. rhythm at a frequency of 1.25 per sec.; (b) 50 per cent. modulation; (c) 25 per cent. modulation; (d) beats formed by two rhythms of $50 \mathrm{c}$. $/ \mathrm{sec}$. and $49 \mathrm{c}$. $/ \mathrm{sec}$. and equal amplitudes; $(e) 49 \mathrm{c}$./sec. reduced to 50 per cent.; $(f) 49 \mathrm{c}$./sec. reduced to 25 per cent.

of the $50 \mathrm{c} / \mathrm{sec}$. rhythm are not resolved the beats and fluctuations in amplitude are clear. From this figure it is seen that when modulation is 100 per cent., (a), and the amplitudes of the two rhythm; are equal, i.e. the beats are of maximal size, $(d)$, the difference in the outlines of a single rhythm modulated in amplitude and the resultant of two waves beating is clear. When, however, the modulation is reduced to 50 per cent. or less, $(b)$ and $(c)$, and the amplitude of one of the beating waves to one-half or less of that of the other $(e)$ and $(f)$, it is no longer possible to distinguish between modulation and beating by the outlines of the waves produced. If the experiment is repeated using frequencies like those that occur regularly in the e.e.g., instead of the unnaturally high one of $50 \mathrm{c}$./ $/ \mathrm{sec}$., discrimination between the two effects is even more difficult. The results of such an experiment using a $10 \mathrm{c}$./ $/ \mathrm{sec}$. rhythm, modulated to varying depths at $2 \cdot 2$ times a second, are shown in Fig. $3(a),(b),(c)$, and beats formed by an $8.5 \mathrm{c}$. $/ \mathrm{sec}$. rhythm of fixed size and a $6 \mathrm{c}$./sec. rhythm of varied size, in Fig. $3(d),(e),(f$. The use of these slow frequencies makes the outlines of the modulated wave and the beats discontinuous, with the result that it is no longer possible to tell the difference between them, even when the modulation is 100 per cent. and the beats are maximal, cf. Fig. $3(a)$ and $(d)$.
A particular instance of the importance of knowing whether a fluctuating rhythm is made up of two other rhythms, or whether it is a single rhythm that is modulated in the brain, is given by the diagnosis of deep tumours of the brain (Walter and Dovey, 1944). Here, a separation has to be made between the 4-7 c./sec. rhythms that often occur in the parietooccipital regions and have already been mentioned, and the faster rhythms that occur farther back in the head. If a residual alpha rhythm of appreciable size is present with the eyes open, and cannot be inhibited by the usual measures, it may completely mask a small 4-7 c./sec. rhythm. Conversely, if the 4-7 c./sec. rhythms are larger than the alpha rhythm, it may be impossible to say whether or not the alpha rhythm has been inhibited on opening the eyes. The record in Fig. 4 (a), illustrates this point in a manner that simulates the e.e.g. more closely than the preceding records. In the resultant of the two rhythms, in line three of the record, it is not possible to count the rate or assess the size of the $8.5 \mathrm{c} . / \mathrm{sec}$. rhythm during the period, corresponding to "eyes open," when it is of small amplitude. When the $8.5 \mathrm{c}$. $/ \mathrm{sec}$. rhythm is large, corresponding to an " eyes closed" period, it is not possible to assess the size or rate of the $6 \mathrm{c}$. $/ \mathrm{sec}$. rhythm.

The effects of over-breathing may be given as another example of the occurrence of masking of 


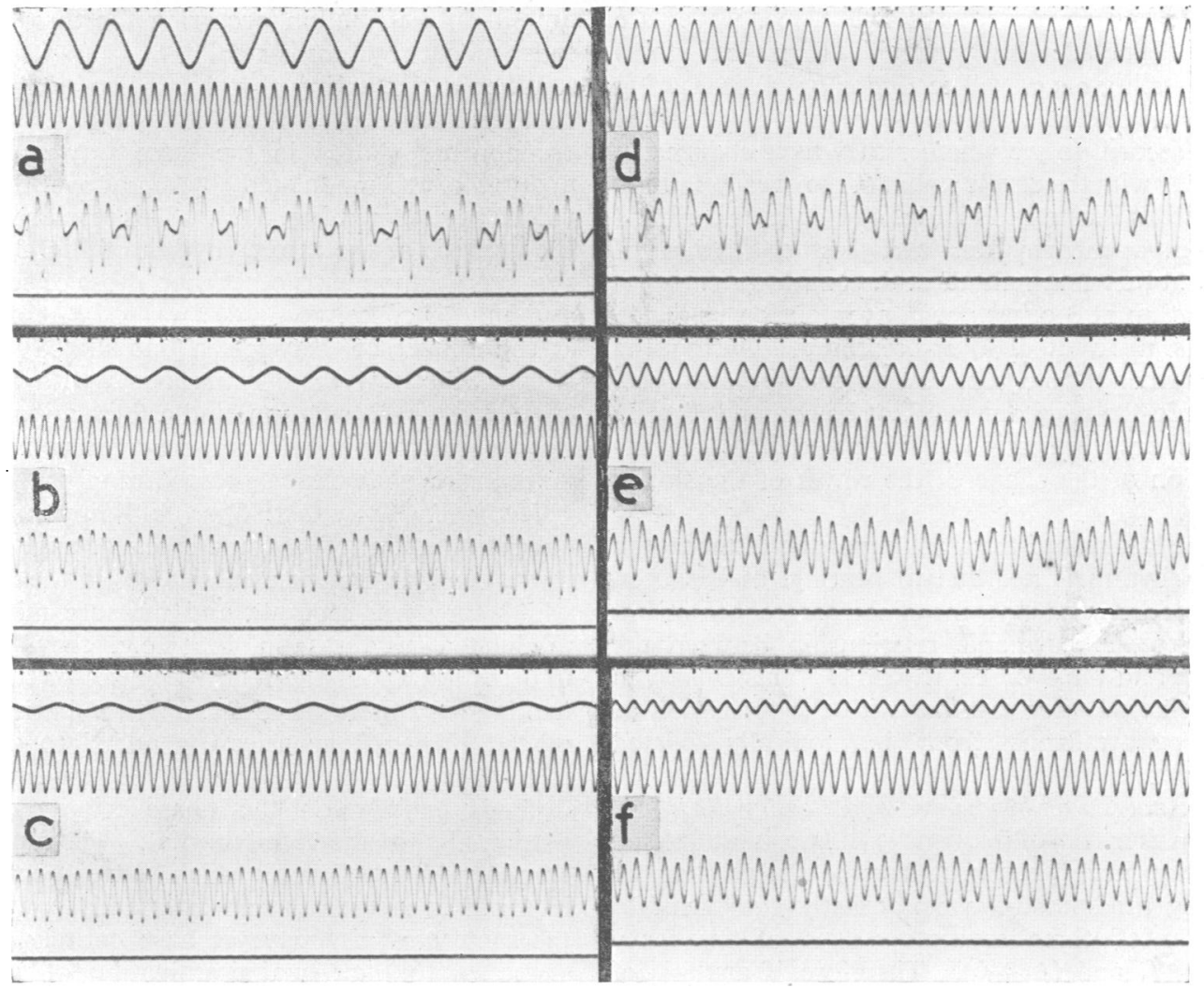

Fig. 3.-(a) 100 per cent. modulation of a $10 \mathrm{c}$. $/ \mathrm{sec}$. rhythm at a frequency of $2.2 \mathrm{c} . / \mathrm{sec}$.; (b) 50 per cent. modulation; (c) 25 per cent. modulation; (d) beats formed between a rhythm of $8.5 \mathrm{c}$. $/ \mathrm{sec}$. and one of $6 \mathrm{c}$. $/ \mathrm{sec}$. of equal amplitude; (e) $6 \mathrm{c}$. $/ \mathrm{sec}$. reduced to 50 per cent; $(f) 6 \mathrm{c}$. $/ \mathrm{sec}$. reduced to 25 per cent.

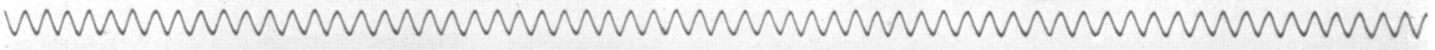

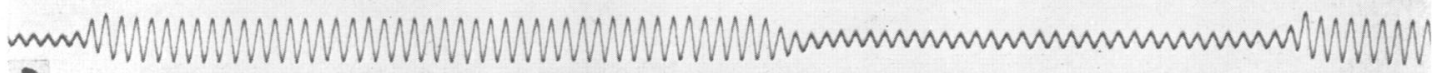 a

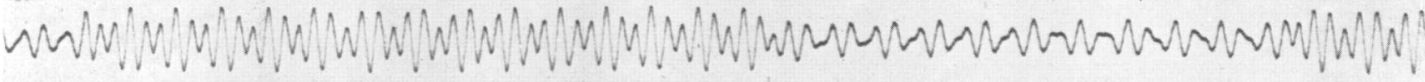

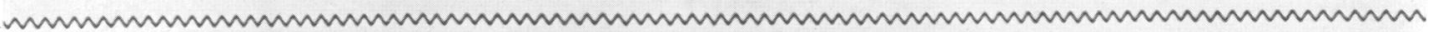

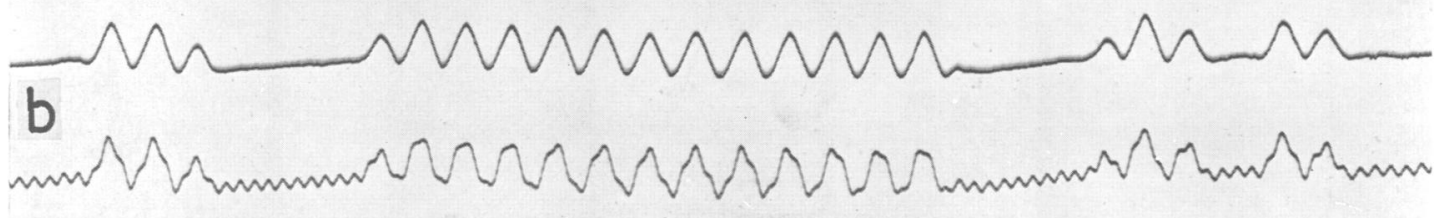

Fig. 4.- (a) Masking of a rhythm at $6 \mathrm{c}$./ $/ \mathrm{sec}$. by one of $8.5 \mathrm{c}$./ $/ \mathrm{sec}$. when the latter is the larger and the convers when the former is the larger; $(b)$ Masking of a rhythm of slightly over $9 \mathrm{c}$./sec. by one of $3 \mathrm{c}$./sec. Note the progressive shift in phase relations indicated by the change in wave shape from square to triangular and back. 
one rhythm by another. When the over-breathing response has developed, the 2-3 c./sec. waves are usually of considerably greater size than the rhythms in the resting e.e.g. The difficulty of measuring any faster rhythms, that have been present in the resting record, to see whether they have changed in size along with the development of the over-breathing response, is illustrated in Fig. $4(b)$. In this record the component rhythms are, first a rhythm of $9 \mathrm{c}$./sec. of fixed amplitude and secondly, a paroxysmal rhythm at $3 \mathrm{c}$./sec., such as might occur in the e.e.g. as a result of over-breathing. During the periods when the $3 \mathrm{c}$. $/ \mathrm{sec}$. waves are large the $9 \mathrm{c}$. $/ \mathrm{sec}$. rhythm in the resultant may not be counted directly and the only indication of its presence is the deformation 'of the shape of the waves of the slower rhythm.

In the last record the masking of the faster by the slower rhythm is not due to beating, as has been described between rhythms of similar frequency, but is due to the harmonic relationship between the $3 \mathrm{c} . / \mathrm{sec}$. and the $9 \mathrm{c}$./sec. rhythms. This effect will be considered later together with the formation of specific wave-forms from harmonically related componenis.

The Analysis of Modulated and Beating Rhythms. -When a rhythm of frequency $f_{1} \mathrm{c}$./sec. is modulated at a frequency $f_{2} \mathrm{c}$./sec. it may be shown that the resulting modulated wave is exactly equivalent to the sum of three separate waves of frequency $\left(f_{1}-f_{2}\right), f_{1}$ and $\left(f_{1}+f_{2}\right)$. Therefore, if analysis of a modulated wave, such as that shown in Fig. $3(a)$, is plotted as a histogram it will show three components: $7.8 \mathrm{c} . / \mathrm{sec}$. $(10-2 \cdot 2), 10 \mathrm{c} . / \mathrm{sec}$., and $12 \cdot 2 \mathrm{c}$./sec. $(10+2 \cdot 2)$, and will appear as in Fig. $5(a)$. The characteristic of such an analysis is the equal size and symmetrical distribution about the rhythm $f_{1}$ of the two subsidiary rhythms $\left(f_{1}-f_{2}\right)$ and $\left(f_{1}+f_{2}\right)$, which are somevimes referred to as " side-

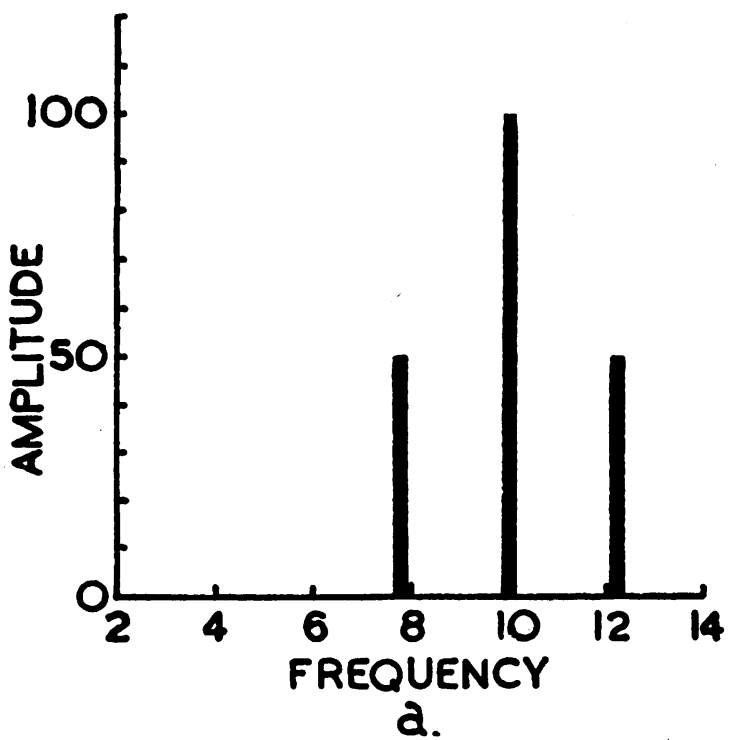

bands." The chance of such a frequency and amplitude distribution occurring for three entırely independent rhythms is small.

When a fluctuating rhythm due to two separate rhythms beating together is analysed, and the components plotted in the same way as for the analysis of the modulated rhythm above, the result will be as shown in Fig. $5(b)$. This result represents the analysis of the mixed rhythm of Fig. $3(d)$ and shows no components other than those of equal size at 6 and $8.5 \mathrm{c} . / \mathrm{sec}$.

The difference between a modulated rhythm, which will show three components with a highly characteristic distribution, and a fluctuating rhythm due to beats, which will show only two components, is therefore made clear by this form of analysis.

Harmonic Series and Specific Waveforms.-The chief limitation of automatic e.e.g. analysis with instruments at present available lies in the fact that whilst they will separate and measure mixed and modulated rhythms in the e.e.g. they give no information about the relative phases of the waves making up these rhythms. When harmonically related higher frequencies are added to a fundamental frequency, the shape of the resulting waves depends entirely on the phase relations of the harmonics to the fundamental. Therefore, two compound waveforms which have components identical in frequency and size, and so will show the same analysis, may yet have entirely different shapes. In the visual examination of an e.e.g. record it is important to know some of the forms a given set of rhythms may produce by phase shifts of the components relative to one another. Such knowledge often makes it possible to deduce the presence in the e.e.g., without the aid of automatic analysis, of frequencies not directly countable.

A series of harmonically related components may produce an infinite variety of waveforms. Only

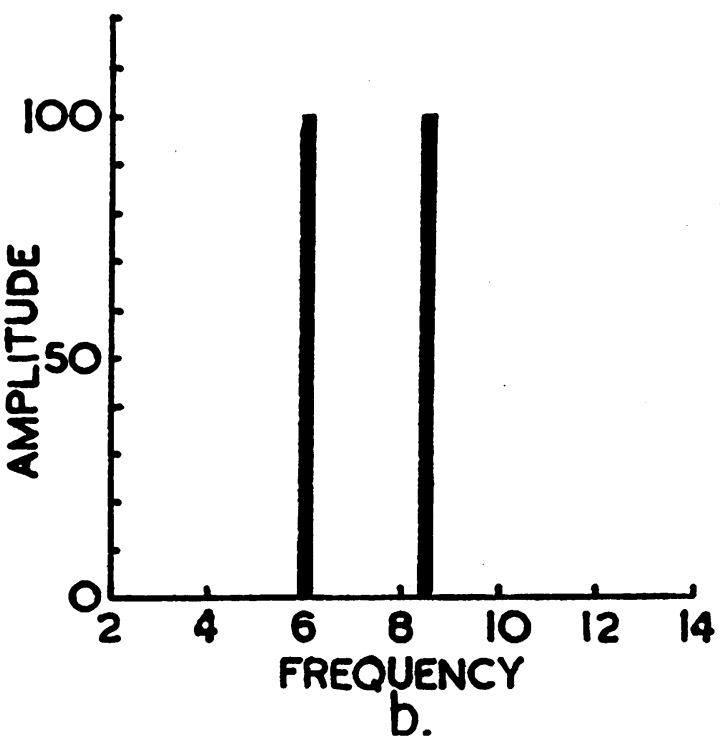

FIG. 5.-(a) Histogram showing components of Fig. $3(a)$. (b) Histogram showing components of Fig. $3(d)$. 
certain shapes common in the e.e.g. need be considered here. How the shapes are built up may be seen most clearly from diagrams covering one cycle of the rhythm.

The first example to be given will be that of mixing two rhythms whose frequencies are in the ratio of $1: 2$, and where the size of the faster rhythm is one-half that of the slower rhythm. The result is shown for two conditions of phase relation; in Fig. 6 the second harmonic starts its first cycle in phase with the fundamental and in Fig. 7 it is advanced in phase by quarter of a cycle or $90^{\circ}$. Though the forms of the resultants are entirely different, their components and, therefore, their analyses are identical.

The combination of waves described above is a common one in the e.e.g. and is more noticeable in the flat topped form, for example, as an asymmetrical or "monophasic" alpha rhythm. In this case it contains components at about $10 \mathrm{c} . / \mathrm{sec}$. and $20 \mathrm{c}$./sec. It occurs also with a fundamental of $2 \cdot 5-3 \mathrm{c}$./sec. in some epileptic conditions. Fig. 10 shows the appearance of this mixture of waves in the form of a continuous rhythm. In this figure the $10 \mathrm{c}$. $/ \mathrm{sec}$. and $20 \mathrm{c}$. $/ \mathrm{sec}$. rhythms are shown separately and then mixed together. The $20 \mathrm{c}$./sec. rhythm is about half as large as the fundamental, with which it is in phase. Fig. 11 shows the same components, but with the second harmonic retarded in phase by $90^{\circ}$. The fact that here the harmonic is retarded in phase causes the bottoms of the waves to be flattened, whereas when it is advanced in phase the tops became flattened, cf. Fig. 7.

The second example to be considered is that of adding to a fundamental rhythm another rhythm of three times its frequency. Fig. 8 shows the effect on the waveform of adding a third harmonic of 50 per cent. of the size of the fundamental and in phase with it at the beginning of the cycle. Fig. 9 shows the addition of the same waves in the same sizes, but with the harmonic advanced in phase by half a cycle or $180^{\circ}$. Here again the two resultants have the same components and analyses, but totally different shapes.

The difference between the wave of Fig. 6, due to the addition of second harmonic, and the wave of Fig. 9, due to the addition of third harmonic, should be noted. The former tends to become saw-toothed in shape as further harmonics are added and is steeper on one side of its peak than the other, while the latter becomes triangular but remains symmetrical on the two sides of its peak. In the same way the wave of Fig. 7 is asymmetrical above and below the baseline whilst that of Fig. 8 is symmetrical in this respect.

Fig. 12 shows, in the form of a continuous rhythm, the effect of adding to a fundamental rhythm of $10 \mathrm{c} . / \mathrm{sec}$. a third harmonic in phase with it: Fig. 13 shows the same addition but with the harmonic $180^{\circ}$ out of phase. Here the contrast in waveform is very clear, as is the possibility of confusion between the waveforms of Fig. 10 and Fig. 13. When both second and third harmonics are added to a funda- mental an even greater variety of waveforms is possible. Fig. 14 shows the effect of combining the waveforms of Fig. 10 and Fig. 13, the lower trace showing the resultant of the two harmonics alone. Here the extreme "spikiness" and the blur along the baseline are the noticeable features. Fig. 15 shows the same components, but as though Figs. 11 and 12 were combined. Here the waveform is quite asymmetrical about the baseline and the spikes are less sharp than in the other combinations. At the recording speed used the blur below the baseline due to the third harmonic is again visible.

If a faster rhythm is not an exact multiple of the frequency of a slower rhythm to which it is added the phase relations of the two rhythms will be constantly changing, and the form of the resultant will pass through the shapes illustrated in a cyclical manner. Thus the waves of Fig. $4(b)$, due to mixing of a $3 \mathrm{c} . / \mathrm{sec}$. rhythm and one of not quite $9 \mathrm{c}$./sec., alternate between the flat-topped form of Fig. 8 and the triangular form of Fig. 9. This effect usually arises in the e.e.g. when the two rhythms that are mixing have some spatial difference in their origins. When the frequencies of two components are accurately locked together and keep constant phase relations it is usually found that no spatial differentiation of their origins can be made. When one of the rhythms drifts slightly in rate with respect to the other, as may be seen from the changes in the shapes of mixed rhythms, a difference in their places of origin should be sought.

Whenever the symmetrical flat-topped or triangular waveforms occur, the presence in the e.e.g. of rhythms at three times the frequency of the fundamental may be deduced. Such forms are common in the rhythms occurring in the e.e.g. in the presence of tumours. They indicate the presence, besides the easily counted $2,2 \cdot 5$, or $3 \mathrm{c}$. $/ \mathrm{sec}$. waves, of components at $6,7 \cdot 5$, or $9 \mathrm{c}$./sec. respectively.

The third example to be shown is the waveform produced when two rhythms with frequencies in the ratio of $2: 3$ are mixed in equal size. Fig. 16 shows two rhythms compounded from these components: (a) in phase, and (b) with the $3 \mathrm{c}$./sec. rhythm advanced in phase by $90^{\circ}$ on the $2 \mathrm{c}$./sec. rhythm. It will be noticed that the effect of a phase shift on the wave form is much less marked than when the frequencies are more widely separated. Also a periodic fluctuation in the size of the wave is beginning to show every other wave. The critical frequency difference at which the eye begins fully to appreciate the presence of beats seems to lie at about a ratio of frequency of $4: 3$. Fig. 17 shows this effect. Here two rhythms with a constant difference of $1 \mathrm{c}$./sec. are shown and the frequencies are progressively reduced from 7 and 6 to 2 and $1 \mathrm{c} . / \mathrm{sec}$.

A striking example of the changes in form that may result from a change in the phase relations of the components of a wave, with their size remaining constant, is given by the synthetic waves of Fig. 18. The basis of the synthesis is an analysis of a wave 


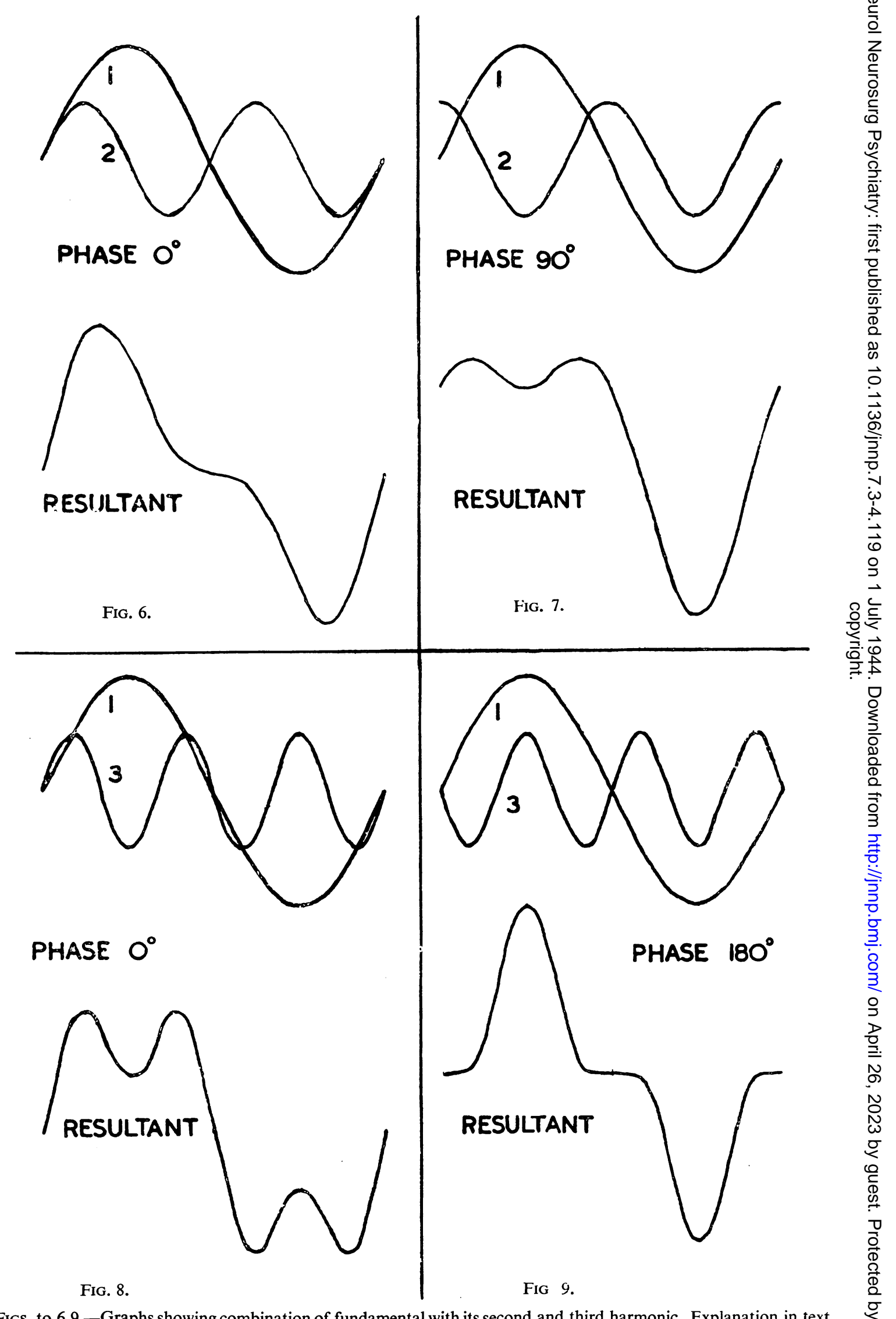

FIGs. to 6 9.-Graphs showing combination of fundamental with its second and third harmonic. Explanation in text. 

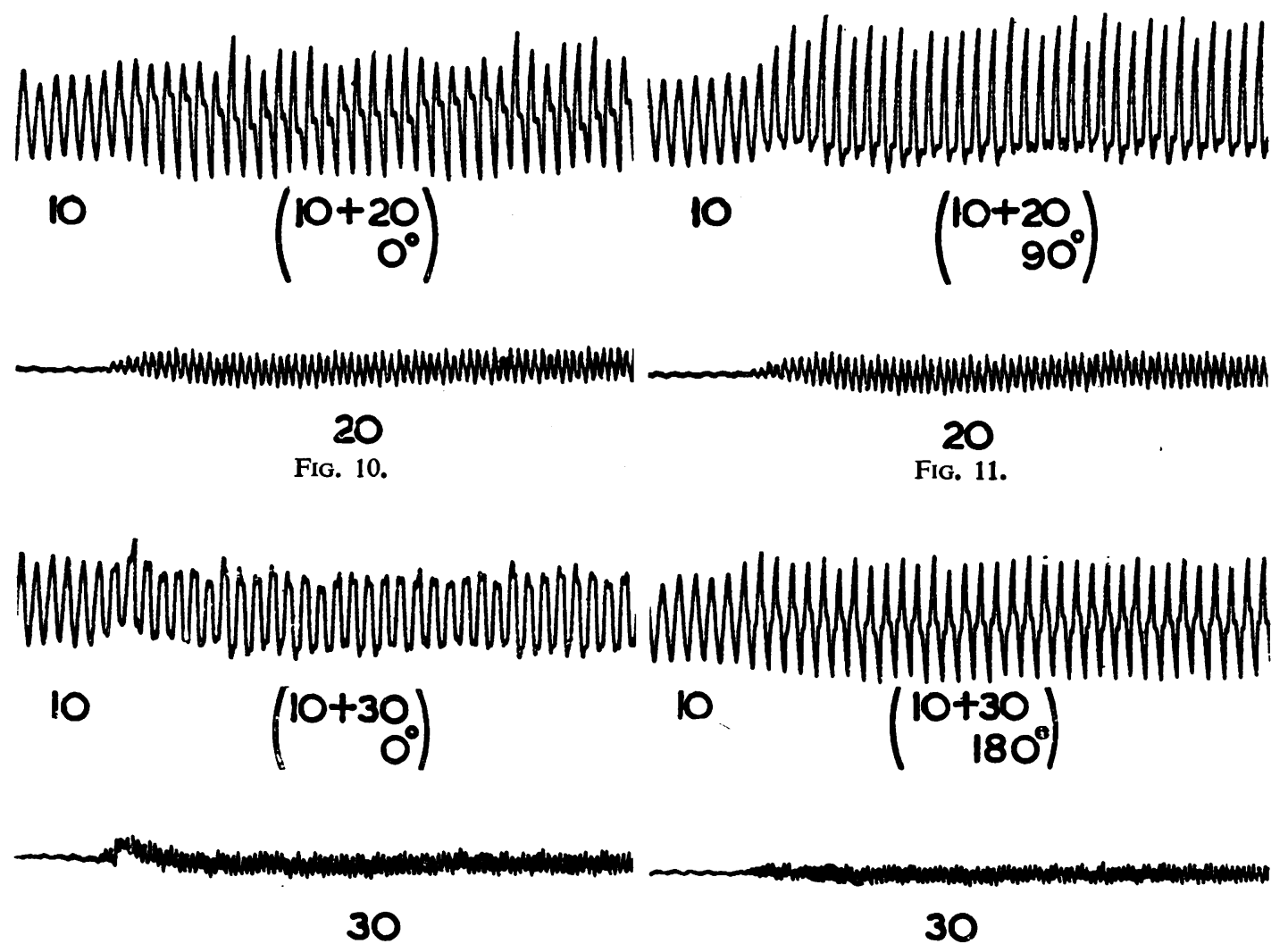

FIG. 12.

FIG. 13.
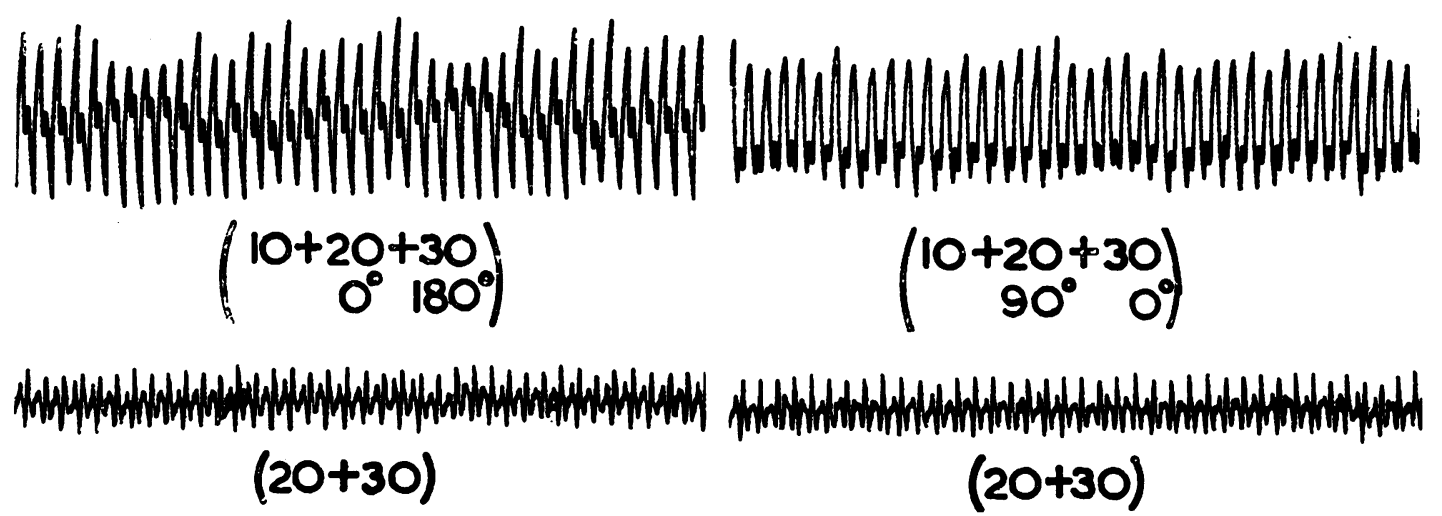

FIG. 14.

FIG. 15.

FIGs. 10 to 15.-Ink-oscillograph tracings of rhythms at 10,20 , and $30 \mathrm{c}$./sec. with various phase relations. The numbers refer to the frequencies present in the trace above and the phase relation of the harmonic to the fundamental in degrees.

and spike discharge from an epileptic subject. The components in the sizes and frequencies indicated in this analysis have been mixed together. Fig. 18 shows first, the spike form produced by the addition of nine frequencies in the ratios of $2,3,4,5,6,7$, 8,9 , and 10, all in phase; secondly, how the addition of the fundamental frequency $180^{\circ}$ out of phase with the other components results in the characteristic wave and spike formation. This arrangement of amplitudes, frequencies, and phases results in the formation of a single spike at the fundamental frequency. Fig. $18(b)$ shows the same components as those responsible for the wave and spike, but out of phase so that although a pattern is produced which repeats at the fundamental frequency, no single spike occurs, and the outline appears more complex. Between the arrows the phase relations of the components are varied at 


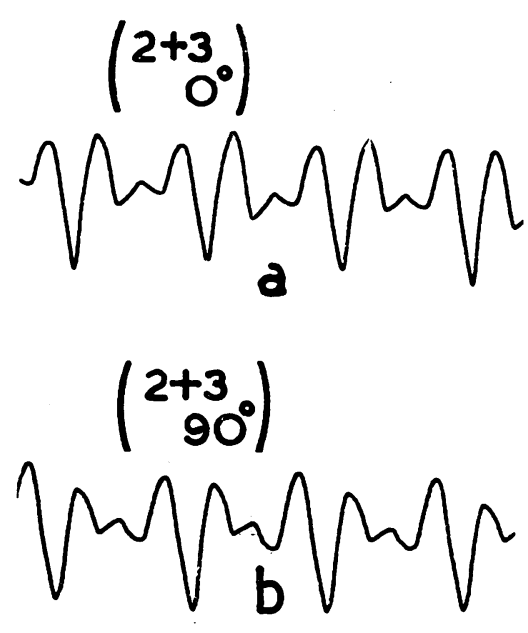

FIG. 16.-(a) Combination of a rhythm at $2 \mathrm{c}$./sec. with one at $3 \mathrm{c} . / \mathrm{sec}$. in phase. (b) The same 90 degrees out of phase.

random and their amplitudes held constant. The waveform here is even more confused and resembles more closely a type of record actually obtained in an epileptic patient between seizures. Fig. $18(c)$ shows the added complexity developed when both phase and amplitude are randomized. Again the resemblance to a natural e.e.g. is even more obvious.

The above examples should make clear the important relation between phase and waveform in complex rhythms. Since instruments for automatic analysis of such rhythms give no information about phase relations, the closest possible association should be maintained between the result of an analysis and the primary record to which it applies.

The value of a wave synthesizer for checking analyses, as well as for demonstration and instruction, is quite considerable.

Separation of Rhythms by Examination Technique. -When an automatic method of analysis is not available it becomes necessary to get the best from every other possible method of separating the components of a complex record. If there are several rhythms occurring in a record and all appear in exactly the same place on the scalp, or in places so close together as to be inseparable by recording from the scalp, separation by examination techniques is not possible. If, however, the rhythms arise in slightly or widely different parts of the brain and appear separable to even a small extent on the scalp, much may be done to simplify the record by suitable electrode placements.

The importance of close electrode placements and bipolar leading for giving the simplest possible record was emphasized by Adrian and Matthews (1934) and Adrian and Yamagiwa (1935). The principle to be used for separating mixed rhythms by electrode placing may best be presented by considering the case, already mentioned, of a parieto-occipital 4-7 c./sec. rhythm and an occipital alpha rhythm.

If the potential gradients produced by the alpha rhythm are plotted, it will be found that they run roughly from the occipital region to the frontal and temporal regions. It is therefore possible to plot out a series of lines at right angles to the radiating gradient lines, such that the points along these lines will have no differences of potential. The gradient lines for a typical alpha rhythm are shown in Fig. $19(a)$, and the equipotential lines corresponding to them in Fig. $19(b)$. If now electrodes placed along the line $\mathrm{R}$, roughly from ear to ear, are used for bipolar recording, the alpha rhythm will either disappear altogether from the record or will be reduced so much that the forms of any other rhythms will not be confused. The 4-7 c./sec. rhythms given as an example usually have an appreciable potential gradient between the parietal and the temporal regions, and so appear unreduced in size with this electrode placement.

The principle of placing electrodes to reduce the size in which particular rhythms will appear in the record may be employed in any part of the head, once the approximate distribution has been worked out for the potential differences against which it is wished to discriminate. It is particularly valuable where the effect of attention, and of opening and closing the eyes, on rhythms other than the alpha rhythm, is to be studied.

An example of the manner in which the suitable placing of electrodes makes it possible partially to separate a complex rhythm into its components is shown in Fig. $20(a)$. The record is of a wave and
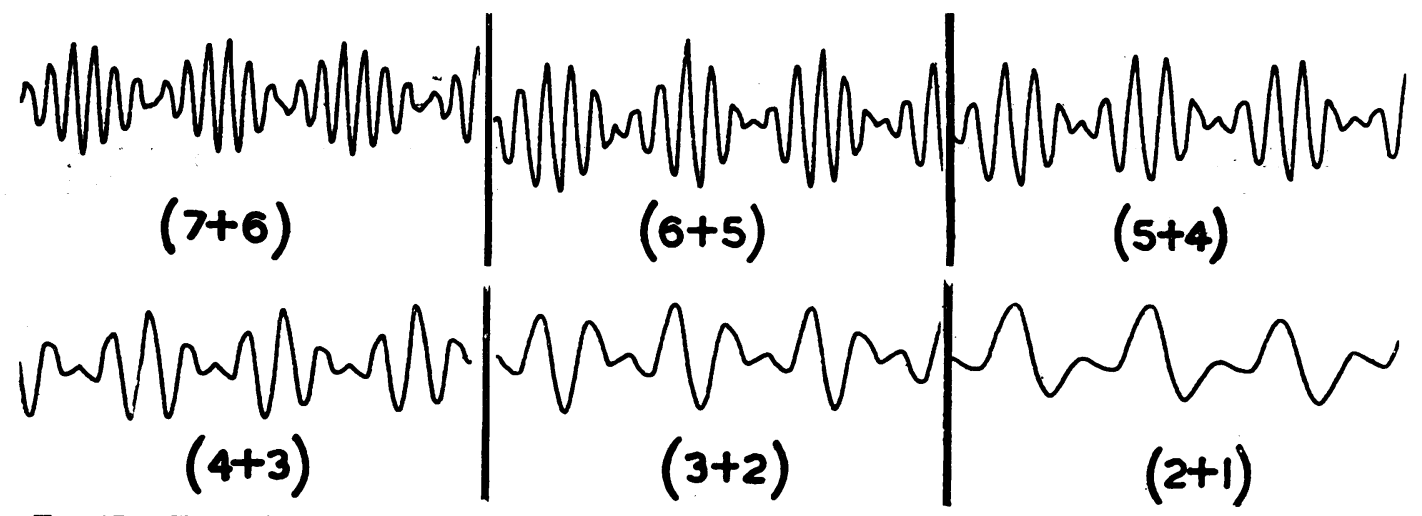

FIG. 17.-Change in the appearance from beats to harmonic distortion as the ratio between two frequencies is increased. 


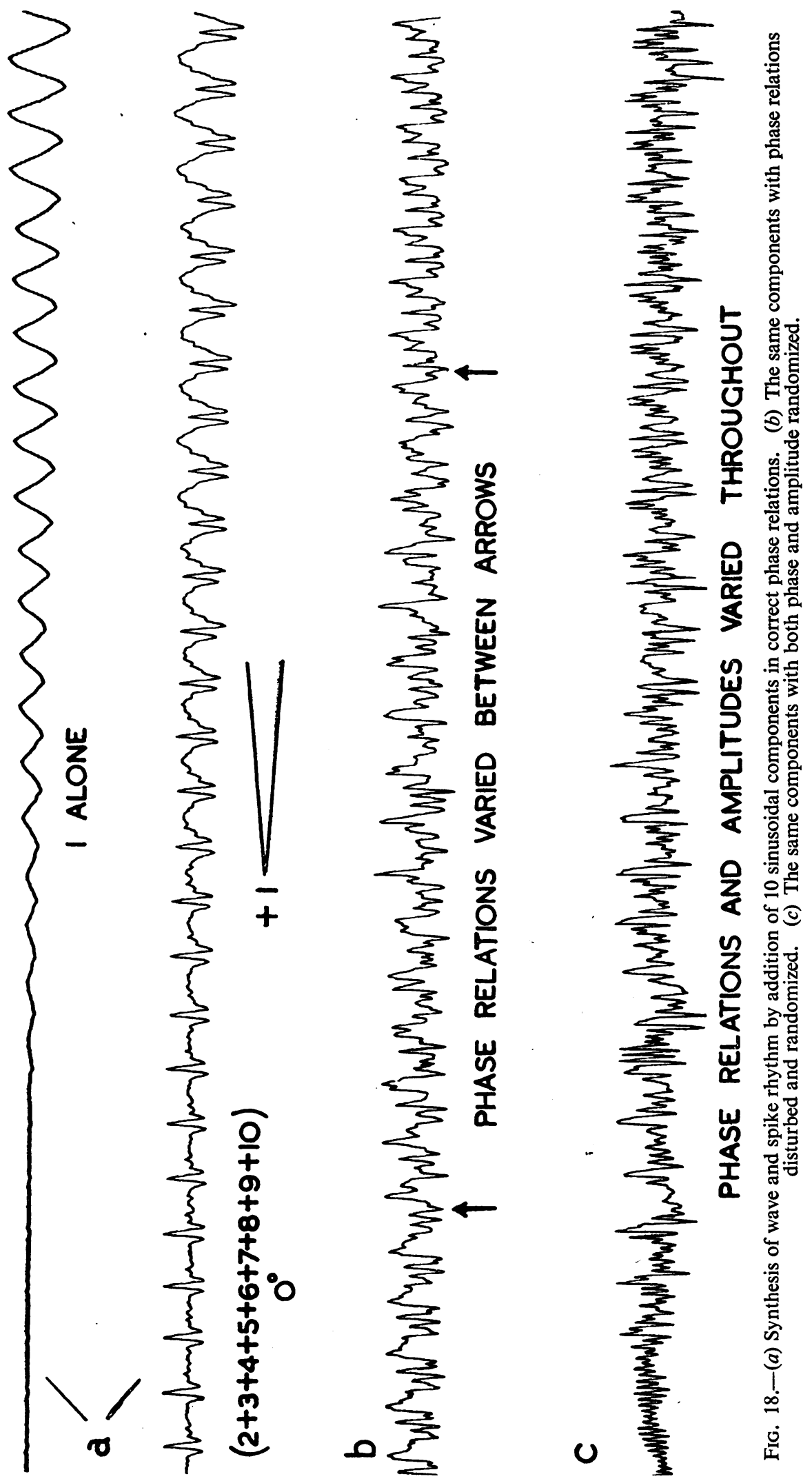




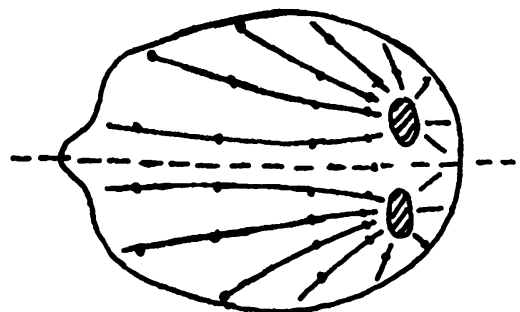

a

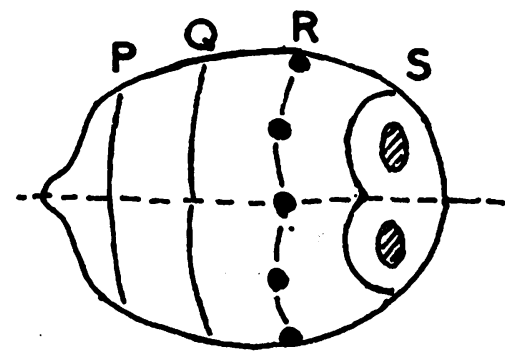

b

Fig. 19.-(a) Potential gradients across the scalp due to two alpha rhythm foci in the occiput. (b) Equipotential lines derived from above.

spike seizure in a subject in whom it is possible to predict accurately the distribution of potential that will occur during such an attack. With the electrodes arranged as shown in the figure the two electrodes of channel three, $\mathbf{R}$ and $\mathrm{S}$, are on an equipotential line with respect to the wave, which therefore practically disappears, but the spike is not reduced in size and therefore has a potential distribution different from that of the wave. Also it is possible to say that, between the second and third, and third and fourth waves of the seizure, the origin of the spike has shifted from below electrode $\mathrm{S}$ to below electrode $R$ and back again, without any corresponding change in the distribution of potential of the wave.

\section{Discussion}

The electroencephalogram is essentially a graph of changes of potential difference with respect to time; in common with any other such graph the waveforms that occur will obey well defined physical principles. The examples of mixed rhythms that are given seem to show quite clearly that in many cases it is not possible to count directly some of the component rhythms. In some cases the importance of component rhythms that are often masked has been demonstrated (Walter and Dovey, 1944), but in others the significance of changes in the masked components has not yet been assessed. If these waves are to be used either as an aid to the distinguishing of abnormality, or in investigation of physiological changes in the e.e.g., every possible means must be used to separate them from the masking rhythms.

When a method of full automatic analysis is not available the first step to be taken should be to obtain as simple a record as is possible. The use of closely spaced bipolar electrodes is of value here as, when two rhythms have spatially different origins, it is possible to discriminate against a large rhythm, and so leave clear for investigation a smaller rhythm it is masking. When the rhythms are not separable by this means the presence of masked rhythms may often be deduced from the forms of the compound rhythm. This method, although it allows the presence of rhythms not directly countable to be described, is of little value as a means of measuring the size of masked rhythms.

Visual examination of an e.e.g. record and direct measurement of the waves in it is reliable so long as the rhythms under investigation are significantly larger than any others present. When, however, the rhythm which is being studied becomes comparable in size with, or smaller than others which are present, a visual examination of the record may be completely misleading unless the masking rhythm can be reduced in size in the record, or some specific shapes occur. For example, in the assessment of the effect of physiological factors on the response of the e.e.g. to over-breathing, any slow waves that may be produced will become countable and measureable as soon as they significantly exceed in size any other rhythms that are present. As soon as this occurs, however, any further changes that may take place in the smaller rhythms will be masked and may not be assessed; Fig. $4(b)$.

If any significance is to be found in changes in rhythms that are masked, either for reasons of size or because of their harmonic relationship to slower rhythms, some form of automatic analysis of the e.e.g. must be used.

In analysing an irregular waveform, such as that of the e.e.g., the record has to be divided up into arbitrary sections corresponding to periods of time. A transient outburst in the e.e.g. may fail to appear significantly in analysis if the duration of the outburst is short in comparison with the length of time chosen for the analysis. The appearance of such an outburst may be highly characteristic and have great significance in interpretation. This is therefore one of the limitations of automatic analysis. The other limitation is the inability of the method to express the phase relations of the components of a compound rhythm.

The importance of the phase relations of the components in a complex wave has been shown in the simple cases of the waves formed from a fundamental and one or two harmonics, and also for the more complicated case of the wave and spike discharge. When a number of rhythms are associated together in a complex wave, and keep constant phase relations, the question arises as to whether or not the components shown in an analysis have any separate existence apart from the fundamental rhythm.

In the case of a fluctuating rhythm, due to two independent rhythms beating together, it is seen that the analysis shows nothing but the two rhythms 

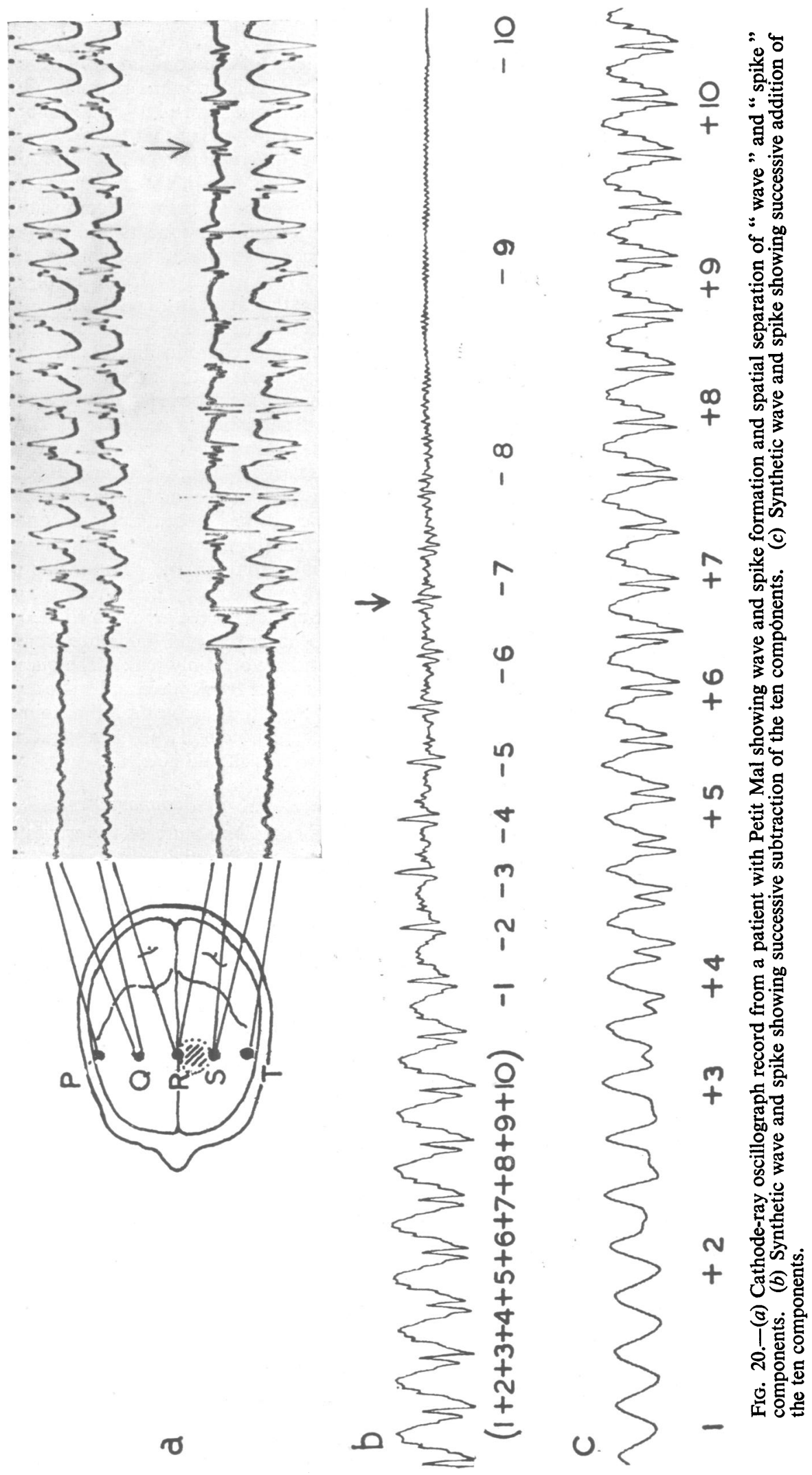
concerned. As the two waves are usually found to have spatially different origins, their appearance in the analysis as two separate components is obviously a correct presentation of the mode of production of the compound wave. The rhythms may therefore be considered as real components that have separate existences. In the case of the modulated rhythm, which shows in analysis three components, the important question to answer is whether these components can be spatially separated or not. If this is not possible by any means whatever, then it seems more likely that the modulation is produced by a fluctuation in the excitability, or degree of synchronization, of a group of cells producing the rhythm, rather than by an exact locking together in the correct ratios of frequency, size, and phase relation of the oscillations of three separate groups of cells. When the three rhythms can be separated spatially, the appearance of modulation is attributable to the existence of three components as distinct entities, and careful location technique will display the components with relatively constant amplitudes.

The possible ambiguity of a three-component analysis illustrates again the essentially complementary relation between the primary records and analyses. In the case where a component in an analysis cannot be shown to have a distinct existence in the primary record, and when there is reason to believe that the component is a modulation product, such a component may be referred to as imaginary.

Where a complex wave shows, in analysis, a fundamental and several harmonics, again some test must be applied to decide whether the harmonics are real or imaginary components. If the harmonically related frequencies cannot be shown to have a separate origin, and their frequencies remain at all times exact multiples of that of the fundamental, it seems likely that the deformation of the wave from the simple sine form is taking place as a result of some inherent limitation in the amplitude with which the group of cells producing it may oscillate. The components will then have no separate existence apart from the fundamental and may be considered as imaginary. When, however, it may be shown that either the harmonics have a different site of origin, even though this difference may be small, or that they do not keep an exact harmonic relation to the fundamental and drift slowly in phase, producing cyclical changes in the form of the compound wave, then it seems justifiable to consider them as being real components and having separate existences.

The question as to what part is played by real and imaginary components becomes of great importance in considering the mode of formation of a complex rhythm, such as that of the wave and spike. Analysis of records from patients liable to wave and spike seizures shows that even between their seizures the component frequencies of the wave and spike discharge are often present in considerable size (Walter, unpublished observations). This observation may suggest that at the time of the seizure a number of separately oscillating systems, which at rest have random phase relations, are becoming locked together in some way to produce the wave and spike discharge. If this is so it implies that some at least of the components in the analysis of the wave and spike rhythm are real and not imaginary. The record shown in Fig. $20(a)$ of a wave and spike rhythm, showing that the spike and the wave may have different potential distributions on the scalp, supports the idea of separately oscillating systems. The spike itself is a complex wave with a large number of components and the record shows the presence of several of these components when the spike has lost its characteristic form at the point marked by the arrow in Fig. $20(a)$. This should be compared with the analogous point similarly marked in the synthetic record in Fig. $20(b)$. In this record the components of the synthetic spike and wave rhythm have been subtracted one by one until, when only the frequencies in the ratio $6,7,8,9$, and 10 remain, the resultant is a periodic wave group without characteristic form. Similarly, Fig. 20 (c) shows how the progressive addition of the constituent frequencies brings about a closer and closer resemblance to the wave and spike formation. Therefore, though some of the components of the wave and spike analysis may be imaginary, others, such as those marked by the arrow in Fig. 20 (a), may be shown to have a spatial difference from the fundamental and are certainly real. The question of how the outbursts of frank wave and spike arise is still an open one, but the value of a full automatic analysis of the e.e.g. potentials in investigating this and other problems should be clear.

\section{Summary}

1. The complex nature of the waves recorded in the e.e.g. is considered.

2. The use of a synthetic method for demonstrating the formation of complex waves, such as occur in the e.e.g., is described.

3. Mixed rhythms of types common in the e.e.g. are synthesized, and the effects on the form of such rhythms of variations in the size and frequency of their components is demonstrated.

4. Certain types of waves which have entirely different compositions are shown to be indistinguishable to the eye.

5. The specific forms that are produced when certain common harmonically related components are added together are shown.

6. The effects on the above forms of the phase relations of the components are demonstrated.

7. The use of the specific forms as a basis for deducing the presence in the e.e.g. of rhythms not obvious to the eye is indicated.

8. Simplification of the e.e.g. by the use of close bjpolar leads is suggested.

9. It is suggested that many of the complex e.e.g. waveforms are due to the simultaneous generation of a number of harmonically related sinusoidal waves.

10. The application of the principles described to e.e.g. examination both with and without automatic analysis is discussed, and the limitations and 
possibilities of visual and automatic analysis of the e.e.g. are considered.

The thanks of one of us (G. D. D.) are due to Mr. Robert Turner, whose generosity made it possible to start this work at the Manchester Royal Infirmary, to the Committee of The David Lewis Colony for facilities to continue it, and to Professor Jefferson, Dr. Ferguson, and Dr. Handley for their encouragement.

\section{REFERENCES}

Adrian, E. D., and Matthews, B. H. C. (1934). J. Physiol., 81, 440.

- , and Yamagiwa, K. (1935). Brain, 58, 323.

Davis, H., Davis, P. A., Loomis, A. L., Harvey, E. N., and Hobart, G. (1938). J. Neurophysiol., 1, 24.

Drohocki, Z. (1938). Comptes Rendus Soc. Biol., 129, 889.
Gasser, H. A., and Erlanger, J. (1927). Amer. J. Physiol., 80, 522 .

Grass, A. M., and Gibbs, F. A. (1938). J. Neurophysiol., 1, 521 .

Kurtz, E. B., and Larsen, M. J. (1935). Elect. Eng., 54, 950.

Livanov, M. M., and Petrova, N. G. (1938). Med. Experimentale (Ukraine), 2, 31.

Parr, G., and Walter, W. G. (1943). J.I.E.E., 90, Part 3, 129.

Rohracher, H. (1937). Pflügers Archiv., 238, 535.

Schaffer, W., and Lubsynski, G. (1931). Proc. Inst. Radio Eng., 19, 1242.

Somerville, J. M. (1944). J. Sci. Instr., 21, 174.

Walter, W. G. (1943a). Electronic Engineering, 16, 9.

- (1943b). Ibid., 16, 236.

and Baldock, G. R. (1944). Ibid. In press. and Dovey, V. J. (1944). J. Neurol. Neurosurg. Psychiat., 7, 57. 